\title{
Effects of a 10-week strength training programme on hand function of patients with hand osteoarthritis
}

\author{
Adebisi I. Hammed ${ }^{1}$, Akindele O. Mukadas ${ }^{2}$ \\ ${ }^{1}$ Department of Human Kinetics and Sports Science, University of Benin, Benin City, Nigeria. \\ ${ }^{2}$ Department of Physiotherapy, Faculty of Allied Health Sciences, Bayero University Kano, Nigeria.
}

\begin{abstract}
The purpose of this study was to evaluate the effectiveness of a 10-week strength training (ST) programme on hand function of patients with hand osteoarthritis (OA). The findings of this study would help clinicians/clinical staff to take ST into consideration in the management of patients with hand OA thereby enhancing clinical outcome. This study was a pre-test, post-test control group design. A total of 30 patients with hand OA participated in the study. Handgrip and pinch strength were measured using electronic hand dynamometer (in $\mathrm{kg}$ ) and mechanical pinch gauge (in $\mathrm{kg}$ ) respectively prior to and following a 10-week ST programme. The amount of handgrip and pinch strength of both hands generated by each participant was used as a quantitative measurement of the development of hand function. Data generated were analyzed using inferential statistics of one way analysis of variance (ANOVA) and the statistical significance was accepted for $p$ value of $<0.05$. The findings of the study showed that the ST programme had significant effects on hand function of patients with hand OA. It was concluded that the ST programme can substantially enhance hand function of patients with hand OA. Therefore, the ST programme should be considered as a key element in the management of patients with hand OA.
\end{abstract}

Keywords. Grip strength, hand function, hand osteoarthritis, strength training.

\section{Introduction}

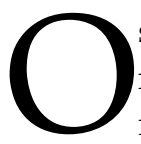
steoarthritis (OA) is a common musculoskeletal disease, traditionally regarded as a degenerative joint condition. It is the most common joint disease and it frequently involves joints of the hands. OA is most prevalent in people over 55 years, although, arthritis in its various forms can start as early as infancy. Hand OA is associated with pain, reduced grip strength, loss of range of motion (ROM), and joint stiffness, leading to impaired hand function and difficulty with daily activities (Bagis et al., 2003). World Health Organization (WHO, 2003) stated that OA is one of the ten most disabling conditions among people above 30 years. It is also suggested that OA will be the fourth leading cause of disability by 2020 (Woolf \& Pfleger, 2003). There are several existing systems for diagnosing osteoarthritis in practice, based on radiographic, pathological or clinical findings as well as the opinion of a physician or specialist (Arden \& Nevitt, 2006), but the most frequently used is the international clinical criteria published by the American College of Rheumatology (ACR) (Altman et al., 1990). According to these criteria, a patient can be classified with hand OA if hand pain, aching, or stiffness for most days of the previous month is present, combined with signs like hard tissue enlargement, swelling, and/or deformity. It should be emphasized that these criteria are primarily developed for classification of patients to be included in clinical trials and observational studies, and not as diagnostic criteria.

Conjointly with the brain, the hand is the most important organ for accomplishing tasks of adaptation, exploration, prehension, precision, perception and manipulation, unique to humans. According to the 
European League Against Rheumatism (EULAR, 2009), the optimal management of hand OA requires both non-pharmacological and pharmacological approaches. Rehabilitative interventions are both nonpharmacological and non-surgical treatments used by therapists in clinical practice to help maintain or regain a person's maximum self-sufficiency and function. These interventions include exercise, splints, heat therapy, electrotherapy, acupuncture, and massage and are recommended for relieving pain and improving hand function, although the level of evidence supporting this recommendation is mainly at the level of expert opinion (Zhang et al., 2009). Moreover, common goals for the treatment of hand OA are pain relief, improved hand strength and range of motion, and reduced stiffness, with an overall goal to improve physical hand function (Kloppenburg, 2007). Consequently, grip and pinch strength seem to decrease with increasing degree of radiographic hand OA (Dominick et al., 2005). Data from the Framingham study on elderly adults show that people with symptomatic hand OA had $10 \%$ reduced grip strength, and more physical impairments when writing and handling small objects than people without symptomatic hand OA (Zhang et al., 2002). On the other hand, strength training (ST), also known as weight training or resistance training has gained popularity in recent years, largely due to its appeal and positive impact on many diverse populations, such as athletic or clinical communities. ST programmes are used to achieve many different goals, such as enhancement of athletic performance, reduction of the risk or rehabilitation of injury and improvement of muscular strength and endurance (American College of Sport Medicine, 2009). According to Ruprai et al., (2015), grip strength and pinch powers are good predictors of total muscular strength and endurance and are important parameters of hand function. The grip and pinch strength measurements are commonly used to evaluate the integrated performances of hand muscles by determining maximal grip and pinch forces that could be produced in one muscular contraction (Norman et al., 2011). Grip and pinch strength measurements are therefore reflections of precisionhandling and are frequently used for quantitative assessment of hand function. Hand strength can be used to determine a treatment, assess nutrition, assess risk of mortality in people with acute or chronic illnesses, as a prognostic factor, and as a marker for general muscle strength and function (Smith et al., 2006).

Furthermore, Stamm et al., (2002) reported that strengthening exercise improves hand function of patients with hand osteoarthritis. Similarly, Rogers \& Wilder (2009) also reported a significant effect of exercise training programme on grip and pinch strength of patients with hand OA. Surprisingly, the exercise programmes that incorporated strengthening exercises failed to find hand strength gains among patients with hand OA yet found an effect on ROM (Lefler \& Armstrong, 2004). In addition, Osteras et al., (2014) asserted that strengthening exercise programme had no significant effects on self-reported hand activity performance in people with hand OA. Likewise, Stukstette et al., (2013) concluded that strengthening exercises had no substantive effects on the grip strength and hand dexterity in patients with hand OA. The improvement of hand function of patients with hand OA following few weeks of ST has not enjoyed a lot of researches over the years. Nevertheless, hand OA is an under-investigated condition with respect to efficacy of various interventions, validated outcome measures and disease impact, compared to OA in other localizations (Kloppenburg et al., 2007). This observed gap in knowledge and research efforts informed the need for the present study. Therefore, the study aimed to determine difference in the hand function of patients with hand OA prior to and following a 10-week ST programme.

\section{Methods}

This study was a pre-test, post-test control group true experimental design. The population of this study included 45 patients between the biological ages of 55 to 73 years who were receiving treatment at the Physiotherapy Department, University of Benin Teaching Hospital, Benin-City, Nigeria.

A total of thirty patients with hand OA in the above mentioned hospital participated in this study. They were recruited using the simple random sampling technique. Balloting without replacement was used to select two-third (2/3) of the population for the study. The names of the patients were written on pieces of paper each and these pieces of paper were put in a bag from where one piece of paper was picked at a time and the name on the piece of paper picked was recorded. 
This process was repeated until the desired sample size was obtained. Thereafter, the recorded names were serialized and systematically assigned randomly into two groups (the experimental and the control groups). Fifteen participants were assigned to the experimental group and the other fifteen to the control group using the same process. The first name in the list was assigned to the experimental group and the second name to the control group, the procedure was continued till the last name in the list was assigned. The selection of the age group is justified by the demographic data suggesting that the highest prevalence of hand OA occurs within this age category compared to younger individuals (Zhang et al., 2002). Patients with hand OA were referred by an orthopedic surgeon or a hand specialist physician who used the inclusion criteria for the classification and reporting of hand OA developed by the ACR. The exclusion criteria were an upper-limb fracture in the past 6 months, neurological disorders, or any other upper-limb problems that could prevent the performance of the experimental tasks.

The research instrument for this study was an adaptation of Sharkely (1990) experimental protocol. The protocol is illustrated as follow:

Table 1

Strength training programme.

\begin{tabular}{ll}
\hline Exercises & Set, Repetition, Rest between sets \\
\hline Bench press & $2^{*}, 8(70 \% 1 \mathrm{RM}), 3$ minutes \\
Military press & $2^{*}, 8(70 \% 1 \mathrm{RM}), 3$ minutes \\
Arm curl & $2^{*}, 8(70 \% 1 \mathrm{RM}), 3$ minutes \\
Latissimus pull & $2^{*}, 8(70 \% 1 \mathrm{RM}), 3$ minutes \\
\hline
\end{tabular}

The following are the test equipment that was used to measure the hand function.

\section{Electronic hand dynamometer}

Hand grip strength of both hands was measured using a Camry Electronic Hand Dynamometer (Model: EH101). It comes with dual scale readout of forces in kilograms and pounds and however, all readings were recorded in kilograms in the present study.

Mechanical pinch gauge

Mechanical pinch gauge was used to measure the three basic pinch tests of both hands including key pinch (lateral pinch) - thumb pad to lateral aspect of middle phalanx of index finger, palmer pinch (chuck pinch) thumb pad to pads of the index and middle fingers, and tip pinch (thumb-index pulp pinch) - thumb tip to index fingertip. It is calibrated in pounds and $\mathrm{kg}$ of force and all readings were equally recorded in kilograms in the present study.

\section{Validity of the instrument}

The test instrument was an adaptation of Sharkely (1990) experimentation. However, the test instrument was validated by experts in physiotherapy as appropriate for the study. The validation was effected at the Outpatients Unit of Physiotherapy Department, University of Benin Teaching Hospital, Benin-City. The following variables were measured: the handgrip and pinch strength as well as hand function of patients with hand OA. It was observed that the use of the facility and procedure were feasible for the conduct of the study. This department was equally served as the project site.

\section{Reliability of the instrument}

A pilot study was conducted to establish the suitability of using the instrument for hand OA patients to which eight (8) hand OA patients, four (4) per group were used. The split-half method of reliability was used in obtaining the data that were subjected to Pearson Product Coefficient of Correlation. Its reliability was calculated to be 0.73 to $0.79(\mathrm{r}=0.73-0.79)$ and it was considered high, therefore, justified the suitability and relevance of using the instrument and protocol for the study.

\section{Method of Data Collection}

The study received ethical approval from the Research Ethics Committee of the hospital to conduct this study. All the participants were recruited consecutively through their hospital files at the Physiotherapy 
Department, University of Benin Teaching Hospital, Benin-City.

\section{ST programme protocol}

Prior to ST programme, a detailed explanation of the test, training programme and the objectives of the study was provided for the participants and then the participants signed a participant's informed consent form before participating in the study and thereafter, the participants were randomly assigned to experimental and control groups. The hand function of both groups were measured before the training and then the participants were subjected to a 10-week ST programme of a frequency of 3 times per week (Monday, Wednesday, and Friday) with each session lasted for 50 minutes at $70 \%$ one-repetition maximum $(70 \% 1 \mathrm{RM})$ consisted of two sets of 8 repetitions for each muscle group with 3 minutes rest between sets. In this way, the protocol consisted of a weekly alteration of the intensity divided into a week of moderate overload (70\% of 1RM, 8 repetitions). The ST programme was purely on upper limbs muscle strengthening that was aimed at improving hand function of the participants. The training programme included bench press, military press, arm curl and latissimus pull, which were performed on a multi weight-lifting machine. Thereafter, hand function of the participants was equally measured after the training.

\section{Grip strength measurement}

To standardize the measurement, the following guidelines were established; the arm positioning followed the American Society of Hand Therapists guidelines (Fess, 1992), with the subject comfortably seated with the shoulder slightly forward and the elbow flexed at a $90^{\circ}$ angle, with the forearm and wrist in a neutral position. A demonstration of how to use the device was first given to each participant by the researcher, to familiarize the participant with the use of the apparatus and to eliminate the element of fear. Alternately, three maximum power gripping efforts were made by each hand of the participant, with threesecond contractions and ten-second rest periods between the attempts and only the best of the three attempts was recorded. Motivation, such as verbal encouragement and competition among group members was used maximally. No assistance of the hand under test was allowed, but facial grimaces and associated movements of the other hand were not discouraged. The device was adjusted for different hand sizes and preferences by adjusting the centre knob and its calibration was also assessed periodically throughout the study.

\section{Pinch strength measurements}

The following guidelines were followed in the measurements of pinch strength; the gauge was "zeroed" before each pinch test by rotating the small curled knob on top of the dial indicator in a counterclockwise direction until it rests against the black pointer at the zero marking. As in grip strength measurement, test instructions and motivation were equally provided.

\section{- Key pinch (lateral pinch)}

The participant comfortably seated or upright, test arm at the side with elbow flexed $90^{\circ}$, palm facing inward, pinch gauge between flexed PIP joint of index finger and thumb, the researcher stood in front of the participant to the side stabilizing the pinch gauge and then had the participant to squeeze, hold and release the pinch gauge (i.e. participant applied pinch force at the pinch groove while holding the pinch gauge between his/her thumb and index fingers). Here, as muscle fatigue begins with the first concentrated effort, a single maximum effort only was recorded.

- Palmer pinch (chuck pinch)

The participant comfortably seated or upright, test arm at the side with elbow flexed $90^{\circ}$, palm facing downward, pinch gauge between thumb and the index and middle fingers, the researcher's position, and duty were the same as in key pinch measurement and also a single maximum effort was recorded.

\section{- Tip pinch (thumb-index pulp pinch)}

Here, the measurement protocol is the same as in palmer pinch (chuck pinch) except that the pinch gauge was between thumb and test finger without the interference of other fingers.

\section{Measurement of hand function}

The present study made use of handgrip strength, key pinch strength, palmer pinch strength and tip pinch strength of both hands as a general quantitative 
measurement of hand function extrapolating the works of Weiss \& Flatt (1971) and Dickson \& Calnan (1972). Therefore, the amount of grip and pinch strength generated by each participant was used as a quantitative measurement of the development of hand function. Similarly, grip and pinch strength testing are commonly used together to evaluate hand function for disability ratings and to assess responses to various forms of therapy. This is because they assess both intrinsic and extrinsic hand muscles (Savas et al., 2007).

\section{Data Analysis}

Data generated were analyzed using descriptive and inferential statistics. The mean and standard deviation were used to summarize the profile of the participants. However, the mean comparison of hand functions for control and experimental groups was analyzed using one-way analysis of variance (ANOVA). Then, Turkey's honesty significant difference post-hoc test was used to identify the source of the difference between the groups. Statistical significance was accepted for $p$ value of $<0.05$. All the analyses were performed using the Statistical Package for the Social Sciences (SPSS) version 22.0.

\section{Results}

The results are presented in Tables 2 - 4. From Table 2, the mean and standard deviation (Mean \pm SD) values of pre-experimental and post-experimental sample group for hand function are $31.02 \pm 3.33$ and $39.10 \pm 2.95$ respectively. Meanwhile, the mean and standard deviation $(\mathrm{M} \pm \mathrm{SD})$ values of pre-control and postcontrol sample group for hand function are 32.74 \pm 3.91 and $32.20 \pm 3.36$ respectively. However, the mean and standard deviation comparisons of the hand function between pre-test and post-test experimental and control group samples are graphically represented in Figures 1.

Table 2

Descriptive statistics of the participants $(n=30)$.

\begin{tabular}{lccccc}
\hline & Mean & SD & SE & Min. & Max. \\
\hline Pre-Experiment & 31.02 & 3.33 & 1.05 & 26.00 & 36.40 \\
Post-experiment & 39.10 & 2.95 & .93 & 33.00 & 42.90 \\
Pre-control & 32.74 & 3.91 & 1.24 & 28.00 & 37.70 \\
Post-control & 32.20 & 3.36 & 1.06 & 28.00 & 38.00 \\
Total & 33.77 & 4.56 & .72 & 26.00 & 42.90 \\
\hline
\end{tabular}

There is no significant difference in the hand function of patients with hand OA prior to and following a 10-week ST programme.

The one-way analysis of variance (ANOVA) conducted to determine the significance of the difference in the hand function prior to and following a 10-week ST programme is presented in Table 3 . The F-value of 11.372 with 3 and 39 degrees of freedom was observed to be statistically significant at 0.05 . Thus, the hypothesis which states that there is no significant difference in the hand function of patients with hand OA prior to and following a10-week ST programme was rejected. This implies that the ST programme had a substantial effect on the hand function of the participants. However, this difference probes the conduct of post-hoc test to identify where the difference lies.

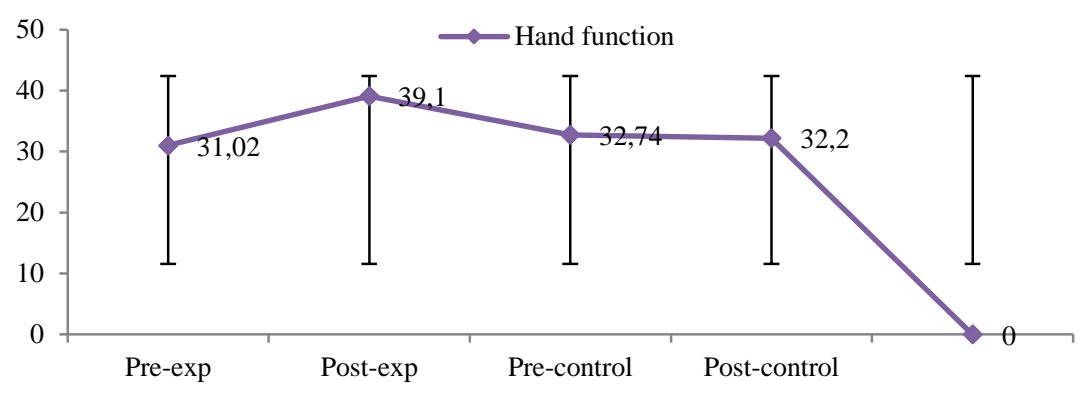

Figure 1. Comparisons of the hand function between pre-test and post-test experimental and control group samples. 
Table 3

Comparison of Analysis of variance (ANOVA) showing the difference in the hand function of the participants.

\begin{tabular}{lccccc}
\hline & Sum of Squares & $\mathrm{df}$ & Mean Square & $\mathrm{F}$ & $\mathrm{p}$ \\
\hline Between Groups & 394.971 & 3 & 131.657 & 11.372 & .000 \\
Within Groups & 416.800 & 36 & 11.578 & & \\
Total & 811.771 & 39 & & & \\
\hline
\end{tabular}

Tukey's HSD test was conducted to determine the difference in variation in the hand function of the participants. There was significant differences between pre- and post-experiment $(\mathrm{p}=0.000)$, pre-control and post-experiment $(p=0.001)$, and post-experiment and post-control $(p=0.000)$. Any significant difference was not found for other comparisons $(\mathrm{p}>0.05)$. This indicates that the entire pair wise mean had variable and thus, the training influenced the variation in the hand function of the participants.

\section{Discussion}

This study showed that the ST programme administered had substantial effect on the participants' hand function. Previous studies have reported similar findings that ST programme had significant effects on hand function of patients with hand OA (Stamm et al., 2002; Rogers \& Wilder, 2009). However, the finding of the present study is not in agreement with Lefler \& Armstrong (2004), Osteras et al., (2014) submissions that ST programme had no significant effect on hand function (hand activity performance) of patients with hand OA. In addition, the study conducted by Stukstette et al., (2013) also concluded that strengthening exercises had no substantive effects on the grip strength and hand dexterity in patients with hand OA, which is also in contrast with the finding of the present study. These contrasting findings might be as a result of variation in study methodology including subject characteristics or differences in measuring instruments of hand function. It could equally be as a result of differences in the content and dosage of exercise programmes. Furthermore, it is also possible that the intensity of the strengthening exercises was insufficient for a change to occur, especially given that increases in strength were not evident.

In addition, the significant improvement in hand function observed in this study can be viewed in two different perspectives. Firstly, the increase in handgrip strength as the root of the initial episode of better hand function because the grip strength reflects the strength generated by the contraction of the various arm and hand muscles involved in the proper functioning of the hand. Secondly, the positive impact of the ST programme on both the intrinsic and extrinsic muscles of the hand and forearm which enable the hand to function smoothly could also explain the better hand function observed in the present study.

However, the limitations of this study were that only the ST was considered because of difficulty in monitoring other activities of the participants, such as prescribed medications and other home programmes and, the chronicity of HOA was not taken into consideration due to inadequate clinical investigations and porosity in the documentation.

\section{Conclusion}

Based on the effects of a 10-week ST programme on hand function of patients with hand OA, it was concluded that the ST programme can substantially improve hand function of patients with hand OA. Therefore, the ST programme is a good training modality for improving hand function of patients with hand OA.

Based on the findings, the following recommendations were made:

1. The ST programme should be considered a key element in the management of hand OA.

2. Hand function measures should be introduced into clinical practice.

\section{References}

Altman R, Alarcon G, Appelrouth, D, Bloch D, Borenstein D, Brandt $\mathrm{K}$. The American College of Rheumatology criteria for the classification and reporting of osteoarthritis of the hand. Arthritis Rheum, 1990; 33(11): 1601-1610. 
American College of Sports Medicine position stand. Progression models in resistance training for healthy adults. Med Science Sports Exercise, 2009; 41(3): 687-708.

Arden N, Nevitt MC. Osteoarthritis: epidemiology. Best Pract Res Clin Rheumatol, 2006; 20(1): 3-25.

Bagis S, Sahin G, Yapici Y, Cimen OB, Erdogan C. The effect of hand osteoarthritis on grip and pinch strength and hand function in postmenopausal women. Clinical Rheumatology, 2003; 22: 420-424.

Dickson RA, Calnan JS. Hand function: A practical method of assessment. British Journal Surgery, 1972; 59: 316-317.

Dominick KL, Jordan JM, Renner JB, Kraus VB. Relationship of radiographic and clinical variables to pinch and grip strength among individuals with osteoarthritis. Arthritis Rheum, 2005; 52(5): 1424-1430.

Fess EE. Grip Strength. American Society of Hand Therapists. 2nd edition, Chicago, 1992.

Kloppenburg, M. Hand osteoarthritis-an increasing need for treatment and rehabilitation. Curr Opin Rheumatol, 2007; 19: 179-183.

Kloppenburg M, Stamm T, Watt I, Kainberger F, Cawston TE, Birrell FN. Research in hand osteoarthritis: time for reappraisal and demand for new strategies. An opinion paper. Ann Rheum Dis, 2007; 66(9): 1157-1161.

Lefler C, Armstrong WJ. Exercise in the treatment of osteoarthritis in the hands of the elderly. Clinical Kinesiology, 2004; 58: 13-17.

Norman K, Stobäus N, Gonzalez MC, Schulzke JD, Pirlich M. Hand grip strength: outcome predictor and marker of nutritional status. Clin Nutr, 2011; 30: 135-142.

Osteras N, Hagen KB, Grotle M, Sand-Svartrud AL, Mowinckel P, Kjeken I. Limited effects of exercises in people with hand osteoarthritis: results from a randomized controlled trial. Osteoarthritis and Cartilage, 2014; 22: 1224-1233.

Rogers MW, Wilder FV. Exercise and Hand Osteoarthritis Symptomatology: A Controlled Crossover Trial. Journal of Hand Therapy, 2009; 22: 10-18.

Ruprai, R.K, Tajpuriya, S.V. \& Mishra, N. Handgrip strength as determinant of upper body strength/physical fitness: a comparative study among individuals performing gymnastics (ring athletes) and gymnasium (power lifters). International Journal of Medical Science and Public Health, 2015; 5: 1- 6 .

Savas S, Koroglu BK, Koyuncuoglu HR, Uzar E, Celik H, Tamer NM. The effects of diabetic related soft tissue hand lessions and the reduced hand strength on functional disability of hand in type 2 diabetic patients. Diabetes Research and Clinical Practice, 2007; 77(1): 77-83.

Smith, T, Martin M, Henry R, Weeks S, Bryant A. Grip Strength in Relation to Overall Strength and Functional Capacity in Very Old and Oldest Old Females. The Haworth Press Inc., 2006: 63-78.

Stamm TA, Machold KP, Sinolen JS, Fischer S, Redlich K, Graninger $\mathrm{W}$. Joint protection and home hand exercises improve hand function in patients with hand osteoarthritis: a randomized controlled trial. Arthritis Rheum, 2002; 47(1): 44-49.

Stukstette MJ, Dekker J, den-Broeder AS, Westeneng JM, Bijlsma JW, van den Ende $\mathrm{CH}$. No evidence for the effectiveness of a multidisciplinary group based treatment program in patients with osteoarthritis of hands on the short term: results of a randomized controlled trial. Osteoarthritis and Cartilage, 2013; 21: 901-910.

Weiss MW, Flatt AE. A pilot study of 198 normal children: Pinch strength and hand size in the growing hand. America Journal of Occupational Therapy, 1971; 25(1): 10-12.

Woolf AD, Pfleger B. Burden of major musculoskeletal conditions. Bull World Health Organ, 2003; 81(9): 646-656.

Zhang W, Doherty M, Leeb BF, Alekseeva L, Arden NK, Bijlsma JW, Dincer F, Dziedzic K, Hauselmann HJ, Kaklamanis P, Kloppenburg M, Lohmander LS, Maheu E, Martin-Mola E, Pavelka K, Punzi L, Reiter S, Smolen J, Verbruggen G, Watt I, Zimmermann-Gorska I. EULAR evidence based recommendations for the diagnosis of hand osteoarthritis: report of a task force of ESCISIT. Ann Rheum Dis, 2009; 68: 8-17.

Zhang Y, Niu J, Kelly-Hayes M, Chaisson CE, Aliabadi P, Felson DT. Prevalence of symptomatic hand osteoarthritis and its impact on functional status among the elderly: The Framingham Study. Am J Epidemiol, 2002; 156(11): 10211027. 